\title{
On free recall and free-recall scoring in immediate memory
}

\author{
ROBERT G. CROWDER, Yale University, New Haven, Conn. \\ 06510
}

Subjects were asked for either free or ordered recall of eight-consonant immediate memory stimuli; ordered recall was scored by both free- and ordered-recall criteria. In terms of total errors, free-recall scoring performance was better than ordered-recall scoring; however, instructed free recall was better than ordered recall scored by a free-recall criterion. In all cases, emission of a redundant prefix increased error frequencies. There was evidence that with regard to the shape of the serial position curve, the instructional set was more important than the scoring criterion.

At least logically, there are two things $S$ must retain in the typical immediate memory task: the identity of the elements presented and the order in which they occurred. A persistent theoretical question has been whether this logical separability is matched by separability of the underlying psychological mechanisms. Crossman (1961) and others have maintained that order and item information are stored independently, Conrad (1965) has argued that in some cases order errors are the consequences of item errors, while associative theories (Wickelgren, 1966) generally take the position that the learning of order relations is an integral part of learning items. Investigators who are sensitive to this issue have often adopted more than one scoring index, usually one that requires correct placement of elements and another based on a "free-recall criterion" (Aaronson, 1968; Moray \& Barnett, 1965; Wickelgren, 1963, 1964). In these studies the free-recall score has been taken from data collected under ordered-recall instructions. To the extent instructions affect acquisition strategies there is the possibility that such measures misrepresent "pure" item information. One purpose of the present study was, therefore, to see whether different results occur when ordered recall is scored by a free-recall criterion as opposed to when the instructions call for free recall in the first place.

The second purpose of the present study was to determine whether the prefix effect in immediate memory (Conrad, 1958; Crowder, 1967) is characteristic of free as well as ordered recall. Research on the prefix effect has indicated that when S must emit a redundant word (such as "zero") between presentation and ordered recall of a series there is a significant impairment in performance, even though the prefix is used over long blocks of trials and never occurs as one of the to-be-remembered elements. With respect to the distinction between order and item information and equally with respect to the ultimate theory of the prefix effect, it is important to know whether, and to what extent, a redundant prefix affects free-recall performance.

\section{METHOD}

Twenty-four paid Yale undergraduates recalled 48 eightconsonant series, each of which was a randomly selected and ordered subset of a basic 12-letter vocabulary (CGHJKLMQRSTX) with rules against alphabetic sequences longer than two and against repetition of a letter in the same serial position on adjacent trials. The four conditions of the experiment were defined by two recall instructions, free and ordered, and two recall conditions, prefix and control. Each S served in all four conditions. Although the 48 series were presented always in the same order, the session was divided into four 12-trial blocks and conditions were administered to different $\mathrm{Ss}$ in different orders such that conditions were balanced against practice effects, sequence effects, and individual stimuli.

The memory stimuli were photographed (all eight letters in a row), mounted on $2 \times 2$-in. slides, and presented by a Kodak Carousel slide projector associated with a Tork 60 -sec timer. The duration of stimulus exposure was $2.5 \mathrm{sec}$, followed by a 17.5 -sec recall interval. The noise of the slide change mechanism served as a warning signal for the next trial. Ss were asked to read the stimulus aloud while it was on the screen and to write their recall on the answer sheets provided. Emission of the prefix element (the word "zero"), when applicable, was vocal. Under free-recall instructions Ss were told to write down as many elements presented in the stimulus series as possible, without regard for order, but under no circumstances to write down more than eight elements. Ordered-recall instructions stipulated left-to-right responding and the leaving of dashes in place of forgotten elements. In all cases the answer sheets had eight spaces in a row opposite the number of each trial.

\section{RESULTS AND DISCUSSION}

The main results are given in Fig. 1, which shows mean recall failures as joint functions of serial position and recall condition; the first panel is for the ordered-recall condition, the last for the free-recall condition, and the center panel represents a scoring of ordered-recall data by a free-recall criterion. In ordered-recall scoring, credit was given only when an element occupied its proper serial position. In free-recall scoring, credit was given for a position if, on the trial in question, the proper element was included somewhere in S's recall; i.e., the abscissa value in Fig. 1 refers to position in presentation and not in recall. Clearly, ordered recall was much harder than free recall in the present study, whether free recall was obtained under ordered- or free-recall instructions; among the 24 Ss there were no exceptions to the former generalization and only one to the latter. Less obvious from the figure, but more interesting, was the finding that free-recall scores were significantly higher when obtained under free-recall instructions than when obtained under orderedrecall instructions. This difference was true of 16 out of the 24 Ss $(p=.076$ by sign test), but a Wilcoxon test showed the two scoring conditions to be significantly different $(T=70.0$, $\mathrm{p}<.05$ ). Thus, scoring ordered-recall data by a free recall criterion gave a biased estimate of Ss' abilities to retain item information from immediate memory strings.

Also evident from Fig. 1 is the finding that a redundant prefix spoken between presentation and recall impaired performance whatever the instructional or scoring procedure

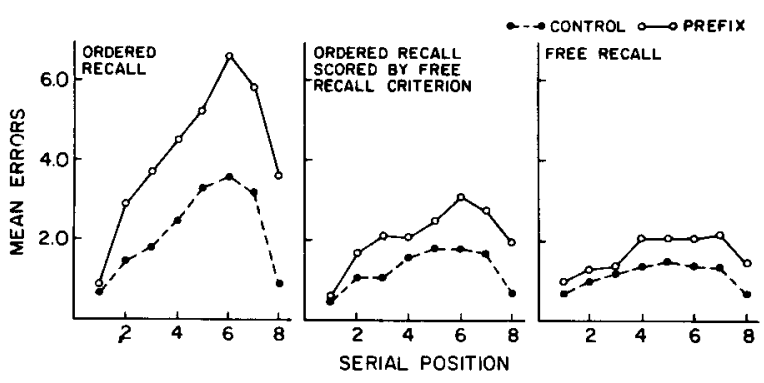

Fig. 1. The relation between errors and serial position for the two recall conditions and the three instructional-scoring conditions. 




Fig. 2. Normalized serial position functions taken from Fig. 1.

( $<<.001$ for each panel of Fig. 1). The prefix effect is therefore clearly not restricted to ordered-recall situations. However, a significant interaction term from an analysis of variance on the ordered- and free-recall conditions revealed that the prefix effect was larger when order information had to be retained than when only item information had to be retained $[F(1,23)=41.6, p<.01]$; therefore, the decrement is not only a loss in item information.

Recent theoretical analyses of recall behavior have shown as much or more concern with the form of the serial position function as with overall performance levels (Aaronson, 1968; Glanzer \& Cunitz, 1966). However, it is difficult to dissociate shape from elevation visually in the data of Fig. 1, so Fig. 2 presents normalized serial position curves, where errors are expressed at each position as proportional to total errors for that condition. In general, the comparison of Figs. 1 and 2 suggests that whereas in total errors (Fig. 1) the method of scoring was more important than the instructional set, in the shape of the serial position curve (Fig. 2) the instructional set was more important than the method of scoring. That is, the center panel of Fig. 2 appears more like the left-hand panel than the right-hand panel. There is no satisfactory single number with which to describe the form of a serial position curve; however, a rough index of the form, more particularly of the skewness of the curve, is the proportion of all errors falling in the first half of the series. Statistical tests of this primacy index across conditions generally supported the claims made above ${ }^{2}$ : When $S$ s had been asked for ordered recall there was no evidence of a difference between orderedand free-recall scoring (14-8 split of nontied $S s, p>.05$, $T=124.5, p>.05$ ). There was more primacy in instructed ordered recall than in instructed free recall (15-7 split of nontied Ss, $p=.067, T=61.5, p<.05$ ). And finally, there was greater primacy when ordered recall was scored by a free-recall criterion than in instructed free recall (17-5 split of nontied Ss, $p=.008, T=66.5, p<.06$ ). Although two of the statistical outcomes are marginal, it appears that with regard to the shape of the serial position function S's set for ordered or free recall is more important than which type of criterion is ultimately applied.

The data in Fig. 2 show that emission of a redundant prefix does not have a large effect on the form of the serial position curve; in no case was there statistical evidence that the prefix affected the primacy index for the three instruction-scoring conditions. However, inspection of the figure suggests that there were substantial differences in the proportion of errors in the eighth position for prefix and control conditions (with, of course, compensatory differences elsewhere). Only in the ordered-recall condition was there strong statistical evidence for a selective prefix effect upon the last serial position; of the 21 nontied Ss in ordered recall only four made relatively more errors on the last serial position in control than in prefix conditions $(p=.004)$. For the other two panels of Fig. 2 , the breakdowns were 14 to $7(p=.095)$ and 11 to $6(p=.166)$, respectively.

\section{REFERENCES}

AARONSON, D. Temporal course of perception in an immediate recall task. Joumal of Experimental Psychology, 1968, 76, 129-140.

CONRAD, R. Accuracy of recall using keyset and telephone dial, and the effects of a prefix digit. Joumal of Applied Psychology, 1958, 42, 285-288.

CONRAD, R. Order error in immediate recall of sequences. Joumal of Verbal Learning \& Verbal Behavior, 1965, 4, 161-169.

CROSSMAN, E. R. F. W. Information and serial order in human immediate memory. In C. E. Cherry (Ed.), Information theory symposium. London: Butterworth, 1961.

CROWDER, R. G. Prefix effects in immediate memory. Canadian Journal of Psy chology, 1967, 21, 450-461.

GLANZER, M., \& CUNITZ, A. R. Two storage mechanisms in free recall. Journal of Verbal Learning \& Verbal Behavior, 1966, 5, 351-360.

MORAY, N., \& BARNETT, T. Stimulus presentation and methods of scoring in short-term memory experiments. Acta Psychologica, 1965, 24, 253-263.

WICKELGREN, W. A. Number of items presented and recalled as determinants of short-term recall. Nature, 1963, 200, 1239-1241.

WICKELGREN, W. A. Size of rehearsal group and short-term memory. Joumal of Experimental Psychology, 1964, 68, 413-419.

WICKELGREN, W. A. Associative intrusions in short-term recall. Joumal of Experimental Psychology, 1966, 72, 853-858. NOTES

1. This research was supported by NSF Grant GB 4066. The assistance of Kathleen A. Cantanese in collecting the data is appreciated.

2 . These differences were evaluated by both two-tailed sign tests and the Wilcoxon matched-pairs signed-ranks test; since the outcomes were not always in perfect agreement, both are reported. 